\title{
New insights into microbial adaptation to extreme saline environments
}

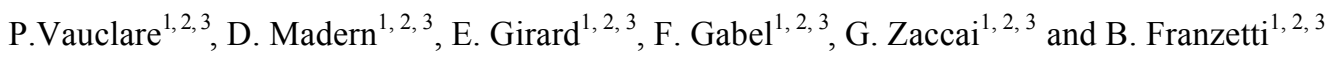 \\ ${ }^{1}$ Univ. Grenoble Alpes, Institut de Biologie Structurale (IBS), F-38027 Grenoble, France \\ ${ }^{2}$ CNRS, IBS, F-38027 Grenoble, France \\ ${ }^{3}$ CEA, DSV, IBS, F-38027 Grenoble, France
}

\begin{abstract}
Extreme halophiles are microorganisms adapted to low water activity living at the upper salt concentration that life can tolerate. We review here recent data that specify the main factors, which determine their peculiar salt-dependent biochemistry. The data suggested that evolution proceeds by stage to modify the molecular dynamics properties of the entire proteome. Extreme halophiles therefore represent tractable models to understand how fast and to what extent microorganisms adapt to environmental changes. Halophiles are also robust organisms, capable to resist multiple stressors. Preliminary studies indicated that they have developed a cellular response specifically aimed to survive when the salt condition fluctuates. Because of these properties halophilic organisms deserve special attention in the search for traces of life on other planets.
\end{abstract}

\section{The extreme biosphere}

Microbes are the most diverse and abundant organisms on earth. A large part of the earth environment is exposed to physico-chemical conditions that, for a long time, were thought to be incompatible with life. The discovery of microorganisms that actually require extreme conditions for optimal growth and efficient metabolism changed this anthropocentric view. Furthermore, environmental microbiology studies have revealed that there are actually very few sterile environments on earth (1). Extremophiles have been identified in all kinds of physico-chemical conditions: high and low temperatures (thermophiles and psychrophiles), pressure (piezophiles or barophiles), $\mathrm{pH}$ (acidophiles and natrophiles) and salt (halophiles) (2). Microbial communities are present in the deep oceans, in soils, in cold and dry deserts, and even in the deep sub-surface (3). Moreover, the explosion in genome sequencing and recent environmental microbiology studies have revealed an unexpected biodiversity in these environments (4). "Extremophiles" are, therefore, widely distributed and represent a large part of the earth biomass. Archaea are the third kingdom of life (5). They are often dominant in extreme environments. However, mesophilic archaea also represent a significant part of non-extreme environmental microbiomes like soils or animal guts (6). The extremophiles often rely on geo-chemical resources as electron donors for their energy metabolisms and represent the core of rich ecosystems $(7,8)$. Extreme environments are also amongst the more dynamic ecosystems on earth. As a consequence, extremophiles possess remarkable resilience and stress resistance capacities. For all these reasons, extremophiles deserve a special interest in planetary sciences and astrobiology and represent tractable model to understand the evolution of life with respect to environmental changes as well as to dissect the cellular stress response (9). 


\section{Extremophilic life adaptation: general concepts and future challenges}

In addition to the preservation of cellular ultrastructure and maintaining genomic integrity, adaptation to extreme conditions implies protection against protein denaturation and/or inactivation, which may be irreversible and lead to cell death (10). Microorganisms have developed various strategies to meet this challenge. Cells may stimulate cellular protection systems such as molecular chaperones and proteases to slow down the deleterious effects of unstable physico-chemical conditions (11) and/or accumulate various types of compatible solutes in their cytosol $(12,13)$. These compounds display extensive interactions with the proteins surface and have stabilizing effects due to a reinforcement of the system enthalpy (14). These cellular responses are widely used to face variations in temperature, salt and pressure. However, all these mechanisms are extremely energy costly and do not permit an optimal functioning of the cells. Proteins are dynamic entities: molecular motions and flexibility are essential to allow biological functions, while resilience or rigidity is required for 3D protein folding and stability (15). The ultimate adaptation to extreme conditions is driven by Darwinian evolution. It consists in the selection of mutations that modify in depth the energy landscape of the proteins in order to prevent their denaturation in extreme physiological conditions, while facilitating motions allowing them to keep their function (16). Adaptation of proteome molecular dynamics has been revealed by neutron scattering in bacteria living from different temperature environments (17). Adaptation to high temperature, for instace, does not modify in depth the overall tri-dimensional fold of proteins but proceeds instead by an increase in the intramolecular interactions, a reduction of the loops and a more hydrophobic core $(18,19)$. Protein surface modifications such as acidification or enrichments in stable aminoacids also contribute to stabilize the macromolecule in extreme conditions. By using neutron spectrometry we have shown that these modifications suffice to modify drastically the molecular dynamic properties of a protein, leading to an increase in its mean resilience (18). The study of the enzymatic, biophysical and structural properties lactate-malate dehydrogenases arising from different organisms has shown that a few hot spots in the protein structure are selected by evolution to maintain an adequate local flexibility in order to permit its biological function (19). Thus, it appears that extremophilic molecular adaptation generates highly contrasted dynamic landscape in proteins. It is important to be aware that, since each protein adopts very different pathways to reach its stable fold, no general statement can be drawn on the detailed mechanisms leading to extremophilic adaptation. Conversely, sensitivity towards physico-chemical stress will vary from one protein to another and the adaptation of a microorganism to extreme condition could therefore be limited by the fragility of a reduced set of proteins or biological functions only. It is expected that transcriptomics and proteomics studies under stress conditions could help to identify these key players in extremophilic adaptation.

Because of the specific structural adaptations of the proteomes, extreme conditions are not perceived as a bona fide stress by extremophiles. On the contrary, extremophiles often elicit a cellular stress response when they are exposed to "normal" conditions such as mild temperatures, low salt, low pressure etc (20). Some of the proteins and metabolites involved are common for all types of physico-chemical parameters, but some specific responses seem to exist in the cases of low salt stress or pressure (21-24). Extreme environments are very unstable, as illustrated by the physico-chemical composition of hydrothermal vent fluids, which is constantly changing. The extremophiles living in their vicinity often have to modify their metabolisms to cope with sharp chemo- and thermo-clines and highly fluctuant, often scarce, nutrient sources $(25,26)$. Thus, high metabolic versatility, efficient stress response systems as well as dormancy mechanisms also represent key aspects of extremophilic adaptation. These aspects of extremophilic adaptation remain poorly explored and represent important new fields of research. A pivotal issue is to specify how fast and to what extent an organism can adapt to changes in environmental conditions. In environmental sciences and exobiology, extremophiles represent unique models to 
understand the drivers of changes leading to an obligatory lifestyle associated with a specific harsh environment and its fluctuations.

\section{Halophiles: model organisms in exobiology and environmental microbiology}

Saline environments are of important environmental significance. About one quarter of the land mass contains salt deposits and saline lakes and seas and oceans represent a large part of the earth's surface (27). Halophilic microbes thrive in nearly saturated brines. They are remarkably tolerant to multiple stresses such as desiccation, temperature, toxic metals, radiation etc (28-32). Halophilic strains have even been shown to survive space conditions $(33,34)$. Extreme halophiles are extremely robust when trapped in fluid inclusions within salt crystals $(35,36)$ and cultures can be inoculated from this material many years after the water evaporated away. Moreover, several studies have reported the existence of viable cells from salt deposits hundreds of million years old (37-39). We have shown that halophilic proteins or RNA embedded in salt crystals were protected from heat denaturation (40). Hypersaline conditions exist in other planets of our solar system. Salt deposits have been formed by evaporation of the primitive oceans on Mars and the oceans lying just beneath the frozen surfaces of Europa and Enceladus, the moons of Jupiter and Saturn are believed to be hypersaline (9,41-44). Terrestrial environments can be quite similar to such planetary conditions. For instance, the Deep Halophilic Anoxic Lakes (DHAL's) located in the depths of the Mediterranean sea combine hypersalinity, anoxia, darkness and high pressure similar to the ones expected bellow the surface of Europa (45). Interestingly, diverse, abundant and metabolically active microbial communities of halophiles were identified in these DHAL brines $(46,47)$. For these reasons, salt sediments or hypersaline waters represent interesting sampling areas in the quest for traces of life on other planets. For long periods, the earth's primitive oceans were also hypersaline (48) and it has been hypothesized that life started in hypersaline conditions. The stabilization of RNA molecules by hypersaline conditions could indeed have played a role in the emergence of life in the "RNA world" scenario. However, it is important to bear in mind that the geological history of our planet is extremely chaotic and that the physico-chemical conditions of the oceans have changed a lot, accompanied by massive species extinctions followed by recolonizations. Moreover, phylogenomics studies of halophilic genomes and the recent advances in understanding halophilic molecular adaptation described below have suggested that salt adaptation could be a rapid, stepwise, process and that the contemporary organisms that thrive in theses environments may have arisen from recent adaptations. Therefore, the statement consisting in saying that contemporary halophilic organisms, or more generally "extremophiles", are "living fossils" is probably ill-suited. It remains that halophilism is one of the most tractable types of adaptation to understand how evolution changes in depth the functional and biophysical properties of individual biological molecules and whole cell metabolism to match environmental constraints. Halophilism represents a unique model to identify cellular mechanisms allowing cells to cope with a natural changing environment due to their unique robustness and their ability to cope with multiple stresses. In order to understand the adaptive strategies of halophilic microbes, it is important to specify the limits of stability/solubility of their proteins and to determine their structural specificities.

Unlike halotolerant microbes that counterbalance the difference in osmotic pressure by accumulating compatible solutes in their cytosol, the extreme halophiles accumulate about $4 \mathrm{M}$ $\mathrm{KCl}$ as osmoprotectant (49-51). This concentration, close to the saturation of $\mathrm{KCl}$, is similar to the external $\mathrm{NaCl}$ concentration and allows to balance the osmotic pressure. As a consequence, in extreme halophiles, all biochemical processes need to function in close to saturated salt solutions, implying the development of specific adaptations. Indeed, hypersalinity represents a major stressor for non-adapted organisms. Above the physical constraint on cellular ultrastructure due to the osmotic pressure, hypersalinity induces aggregation in unadapted proteins and limits enzyme activity. Protein solvation, i.e. the interaction of water molecules and ions with 
polypeptide chains in solution, is essential for protein folding and provides a lubricant for molecular dynamics necessary for activity (15). Liquid water is essential for life, and life cannot exists bellow a critical water activity (52). Halophiles are remarkable because they thrive on the edge of the know limits of water activity. In non-adapted systems, such conditions promote proteins aggregation, precipitation, and denaturation, thus reducing the activity of most nonextremophile enzymes. On the contrary, most halophilic proteins display an obligatory requirement for the high salt conditions to be active and stable and, in many cases, low salt conditions result in enzyme inactivation and precipitation $(53,54)$. It is assumed that it is for this reason that the halophilic organisms must maintain a high cytoplasmic salt concentration.

\section{Water in cells}

Water constitutes the intracellular matrix in which biological molecules interact and its dynamic state in cells has been the subject of controversy until neutron scattering experiments provided direct measurements of water diffusion on the atomic scale in living samples of different cell types. Water dynamics on an atomic length scale has been measured in vivo in the cytoplasm of Escherichia coli in experimental timescales covering motions from pure water to interfacial water. In contrast to the widespread opinion that all cellular water is 'tamed' by macromolecular confinement, the data established that water diffusion within the bacteria is similar to that of bulk water at physiological temperature with an about $10 \%$ contribution from solvation shell water (55). A similar conclusion was reached from experiments on human red blood cells (56). Neutron scattering experiments on water diffusion in the extreme halophile Haloarcula marismortui, in contrast, revealed a water fraction with significantly slower diffusion even than solvation shell water (57), while, as was shown by NMR it maintained bulk-like rotational diffusion (58). The mechanisms behind this slow fraction in the Dead Sea halophile are not yet understood. A speculation that it involves a strong and specific $\mathrm{K}^{+}$ion/water interaction with macromolecular carbonyl groups is currently being tested.

\section{Halophilic protein adaptation}

Genomic analyses were performed in order to probe the mechanisms of halophilic adaptation at the molecular level (59). They confirmed that halophilic proteomes are dominated by highly acidic proteins with an average $\mathrm{pI}$ of 4.2. The examination of available halophilic protein structures showed that the negatively charged residues are clustered on the protein surface (60). This observation is consistent with the solvation model proposed by Zaccai and co-workers in which surface bound hydrated salt ions contribute to protein stabilisation and solubility in the supersaline conditions (54). In addition it has been proposed that strong inter-subunit and intra ionic interactions also contribute importantly to the folding of halophilic proteins (61). Thus, both stabilizing interactions between salt ions and obligatory halophilic proteins behavior are believed to be mandatory to retain biochemical function in a salt saturated cytoplasm. This view has been challenged recently by the discovery of non-halophilic strains that possess acidic proteomes (62) and by the work comparing the enzymatic, biophysical and structural properties of the lactate-malate dehydrogenases enzymes from different halophilic strains. In particular, the structural and enzymatic study of the MalDH enzyme from Salinibacter ruber, a halophilic bacterium (57), showed that an adapted enzyme that possesses high activity and solubility under multimolar salt conditions can remain folded and active under low salt conditions $(63,64)$. The $\mathrm{X}$-ray crystallographic structure of the protein revealed a reduced set of adaptive traits. The protein displays a typical acidic surface, solvent exposed and buried hydrophobic surfaces similar to typical halo-adapted enzymes, but it lacks intersubunit ion binding sites. This suggests a stepwise evolutionary pathway characterized by the incremental addition of modifications that lead to an obligatory salt dependence for protein folding and activity. In the case of halophiles, the environmental adaptation is therefore more subtle than the simple modification of the protein surface and is the consequence of an adaptive process from non-halophilic to halophilic organisms. 


\section{Cellular responses to fluctuating salt environments}

Halophilic microbes stop growing when the environmental conditions drop bellow a specific salt concentration. This concentration varies from one strain to another. For the extreme halophilic archaea $H$. salinarum and $H$. marismortui that possess cultivation salt optima above $4 \mathrm{M} \mathrm{NaCl}$ the lower limit is around $2.5 \mathrm{M}$. Below this value the cell mortality rate increases rapidly, probably due to the effect of osmotic pressure on membranes and because of a low intracellular salt concentration, which is believed to be incompatible with the salt-dependent protein stabilization process that we described for the halophilic lactate-malate deshydrogenase enzyme from $H$. marismortui, an extreme halophilic archaeon from the Dead Sea (65). However, low salt stresses occur frequently in the natural environment in which halophiles thrive. Rain, flooding, evaporation and tidal effects often modify drastically the environmental salt concentration. Saltdependent microbes must therefore have developed survival strategies to cope with this challenge.

In order to identifiy the cytosolic perturbations associated with low salt stress, we first studied the modifications of the internal salt concentration after moderately low salt stress in H. salinarum, a model extreme halophilic archaeon. The study showed that the high internal $\mathrm{K}^{+}$and $\mathrm{Na}^{+}$ concentrations cannot be maintained for long periods. For example, a low salt shock from 4.2 to $2.5 \mathrm{M}$ the internal $\mathrm{K}^{+}$concentration dropped down to $1.75 \mathrm{M}$ after a $15 \mathrm{~min}$ lag period (Marty et al, unpublished). Since significant amounts of $\mathrm{K}^{+}$still remained accumulated in the cytosol, one can assume that the energy-dependent $\mathrm{Na} / \mathrm{K}$ pumps are still active and that the cells maintained a minimal metabolic activity. This is surprising since according to the in vitro data obtained on halophilic enzymes the measured intracellular salt concentration after a low salt stress is not compatible with a long-term stability and functioning of the proteins.

As for other types of extreme adaptation, the salt-dependent stability of a specific protein can vary a lot from one protein to the other. It all depends on the types of molecular interactions that one protein uses to adopt and stabilize its 3D fold and to maintain a flexibility to allow its function. In addition, the cytosolic conditions are far different from the one used in vitro to study protein activity and stability. Proteins are not randomly distributed within the cells, they display extensive physical interactions with partners and the natural ligands and products of the enzymatic reactions influences a lot the stability of the proteins. Also, the intracellular protein concentration has been estimated to be $360 \mathrm{mg} / \mathrm{ml}$ in the cytosol of E. coli. Such molecular crowning may also greatly contribute to protein stability. Salt effects should be measured at the level of the whole proteome. There are few techniques allowing to study the folding state of proteins in a living organism. Neutron scattering is a method of choice to probe protein dynamics (66). Neutrons are scattered by atomic nuclei to provide information on the momentum and energy of their motions. The probed length and time scales are defined by the scattering vector $(Q)$ range and energy resolution of the experiment, respectively. By suitably choosing these values, it has been possible to observe average internal dynamics of an entire protein population, in vivo, without the requirement for specific protein labeling. The experiments are based on 'elastic temperature scans', in which neutrons scattered in a very narrow energy window around the energy of the incident beam are observed as a function of sample temperature. The results of the experiment are the mean square displacement (MSD) and an effective force constant $(<k>)$ for the atomic motions, on the chosen length and time scales. These experimental parameters inform on the mean flexibility and resilience of the protein structures, respectively (15). The resilience is with respect to the free energy landscape, i.e. it includes both structural rigidity (the enthalpic term due to internal forces) and conformational sampling (the entropic term). The neutron length-time window is well suited to examine global protein dynamics parameters associated with environmental adaptation and the motions associated to protein folding/unfolding. These studies showed that a mean square displacement (MSD) and an effective force constant $(\langle k\rangle)$ can be extracted from the diffusion signal of freshly harvested living cells. In the case of thermal adaptation this approach was used to demonstrate that 
evolution modifies in depth the dynamics properties of thermophilic cells, with resilience values being one order of magnitude higher than the ones found in mesophiles (17). Since the neutron signal is dominated by proteins, we proposed that the measured resilience (rigidification) reflects the structural adaptation of the thermophilic proteome. We used the same technique to study the molecular dynamic properties of the proteomes of extremes halophiles. The resilience values were found to be higher than those measured for hyperthermophilic strains (Ms in preparation). This apparent rigidification of the proteome is likely to be due to the extensive interaction of the salt ions with the proteins structures rather than to a peculiar composition of the cytosol such as the presence of compatible solutes. This interpretation is supported by measurements on total protein extracts showing the same differences in the molecular dynamics state by comparison with mesophilic extracts (unpublished data). The experiments indicated that a large part of the $H$. salinarum proteome obey to the salt-dependent solvation model described, in vitro, for a few isolated proteins (Ms in preparation). We then studied the effects of a decrease in the low salt stress on the molecular dynamics state of the halophilic cytosolic proteins. There are few studies on the effect of environmental stresses on the proteome state, in vivo. In order to assess if neutrons could be used to detect molecular perturbations under stress conditions, we first studied the effect of temperature shock on $H$. salinarum cells. These archaea also represent good models for thermal stress response since they can tolerate exposition to temperatures up to $60-65^{\circ} \mathrm{C}$. We developed imaging techniques to measure cell integrity in order to make sure that the stressed cells remained alive during the neutron spectrometry experiments. In the scattering vector range that is used to measure intramolecular motions, the signal was very much perturbed, indicating the presence of highly disordered structures in the cytosol of stressed microbes. When using another scattering vector range it was possible to calculate MSD and resilience values under stress conditions. The measured perturbations corresponded to larger molecular motions and increased flexibility such as those involved in unfolding processes. The perturbations were found to be proportional to the intensity of the stress imposed to the cells. This work demonstrated for the first time that neutron spectroscopy represents a tool to measure the effects of environmental stresses the proteome folding state (67). We used this approach to study the effects of salt stress on $H$. salinarum and $H$. volcanii cells. Microbiology controls were carefully performed to determine the viability limits under various salt deprivation conditions. Important effects on the proteome dynamic states were already detected when the external $\mathrm{NaCl}$ concentration dropped below $3 \mathrm{M}$. Interestingly the measured alterations were quite similar to the ones induced by a heat shock. This suggested that a decrease in the intracellular $\mathrm{K}^{+}$concentration affects the protein folding state as an important temperature elevation. We concluded that, even a moderate drop in extracellular salt conditions represents a major stressor for halophilic cells (Ms in preparation).

The biophysical experiments suggested that cellular responses must exist to protect the halophilic proteomes against the deleterious effects of salt deprivation. Comparative genomic analysis revealed a common unique halophilic protein core of 800 proteins and indicated that a third of the genes encode for proteins with no significant matches in to the databases (59). In $H$. salinarum, whole genome DNA microarray experiments showed that a moderate decrease in salt concentration modifies the expression of genes encoding for potassium and phosphate transporters as well as peptide transporters (23). This suggests that under moderate salt deprivation, a first level of response consists in minimizing the loss of ions. Interestingly, in halophilic strains, many of the genes encoding for thermal stress response proteins are also induced under low salt conditions, thus confirming that low salt represents a physico-chemical stressor (68). We studied the accumulation of specific proteins in $H$. marismortui and $H$. salinarum cells exposed to prolonged low salt stress conditions, similar to the one we used for neutrons experiments. We specifically searched for large complexes in the hydrophobic fractions of the total cytosolic extracts of $H$. salinarum and H. marismortui. These studies revealed that the type II chaperonin complex, called "thermosome" in archaea, is strongly accumulated within cells exposed to low salt conditions (68-70). Gene expression studies indicated that the heat shock promoter of the thermosome is also strongly salt regulated, suggesting that the low salt and 
the heat and salt stressors use similar, if not identical, transcriptional regulators. A novel type of chaperonin complex called Ssp45 was also identified (24). The 450-kDa complex is a ring shaped molecular edifice comprising about 10 subunits. The complex displays $\mathrm{Mg}^{2+}$ dependent ATPase activity that is strongly stimulated by the presence of $\mathrm{K}^{+}$ions. In vitro, it binds to proteins in low salt conditions and slows down their unfolding. The protein is rapidly accumulated the halophilic cells shortly after exposure to a lower salt concentration. Interestingly, searches in databases did not allow the identification of SsP45 homologues in nonhalophilic archaeal strains.

Intracellular proteolysis is a pivotal cellular function for environmental adpatation. The rapid destruction or essential proteins, such as regulatory factors or key enzymes, is extensively exploited by the cells for the fine-tuning of many cellular functions such as cell division or metabolism. Protein and peptide hydrolysis also contribute to the energy metabolism in heterotrophic strains or under starvation conditions. In the protein quality control function, the chaperone machinery rescuese disabled proteins that are generated by translational errors, mutations, aging and environmental stresses (71). However, in most cases the chaperoning process fails and the role of chaperones is rather to create a pool of substrates for proteolysis before the disabled proteins could reach a completely irreversible unfolded state that would lead to their aggregation in the cytosol. Ultimately, some chaperones such as the Hsp100 can work as anti-chaperones (72). They use ATP hydrolysis to unfold the polypeptide chains. The process is induced when they are in contact with protease complexes such as the $20 \mathrm{~S}$ proteasome (73). Intracellular proteolysis is therefore essential in environmental stress response and extremophilic adaptation. We measured proteases activities in fractionated extracts of $H$. salinarum, under various physiological conditions. These studies showed that endopeptidases and exopeptidases activities are not regulated in the same way. When looking specifically to the low salt response, we found that the exopeptidases activities decrease while the endopeptidases are strongly stimulated. In particular, the measured intra cellular proteasomes activities were found to be induced shortly after salt dilution (69). The transcriptional and proteomic study of the salt regulation of the $H$. salinarum proteasome system showed that the ATPase regulatory subunits of the proteasome (PAN) were rapidly induced after salt shock, while the proteasome core intracellular accumulation occurred later (69). We tested different proteasome inhibitors in various physico-chemical conditions. These studies showed that halophilic cells remain alive and can still divide with a strongly disabled proteasome function, even under salt-stress conditions. We measured the intracellular protease activities in fractionated cell extracts and, under salt stress conditions and identified compensatory proteolytic activities that are not present in untreated cells (unpublished). Thus, other proteolytic activities may alleviate proteasome defects and represent potential novel proteolytic pathways that still remain to be characterized.

\section{Conclusions}

The recent studies reviewed here demonstrated that extreme halophiles are highly responsive to the salt environments. This work lays the foundation for further investigations that will lead to a better understanding of the extraordinary capacity of extreme halophiles to cope with changing environments and may explain their presence in a broad range of environments such as soils, animal gut, deserts and possibly other planets. The dissection of the stepwise evolutionary process leading to protein adaptation shall indicate how fast microorganisms adapt to the salt constraint. Preliminary data were obtained suggesting that, despite the deleterious effects of hyposaline conditions on the proteome folding state, halophiles can survive for long periods in low salt conditions. Transcriptomics, proteomics and metabolomics analyses should reveal the physiological mechanisms involved this dormancy process and should reveal the molecular mechanisms underlying the broad dispersion of halophilic microbes. 


\section{Acknowledgements}

This work has been supported by the CNRS interdisciplinary program « Environnement Planétaire et Origine du Vivant » (EPOV).

\section{References}

1. Akondi, K. B., and Lakshmi, V. V. (2013) Omics : a journal of integrative biology 17, 6170

2. Pikuta, E. V., Hoover, R. B., and Tang, J. (2007) Critical reviews in microbiology 33, 183209

3. Stokke, R., Roalkvam, I., Lanzen, A., Haflidason, H., and Steen, I. H. (2012) Environ Microbiol 14, 1333-1346

4. Ferrer, M., Golyshina, O., Beloqui, A., and Golyshin, P. N. (2007) Curr Opin Microbiol 10, 207-214

5. Gribaldo, S., Forterre, P., and Brochier-Armanet, C. (2011) Research in microbiology 162, $1-4$

6. Reeve, J. N., and Schleper, C. (2011) Curr Opin Microbiol 14, 271-273

7. Berg, I. A., Kockelkorn, D., Ramos-Vera, W. H., Say, R. F., Zarzycki, J., Hugler, M., Alber, B. E., and Fuchs, G. (2010) Nature reviews 8, 447-460

8. Sato, T., and Atomi, H. (2011) Curr Opin Microbiol 14, 307-314

9. Shapiro, R., and Schulze-Makuch, D. (2009) Astrobiology 9, 335-343

10. Ellis, R. J., and Minton, A. P. (2006) Biol Chem 387, 485-497

11. Goldberg, A. L. (2003) Nature 426, 895-899

12. Benaroudj, N., Lee, D. H., and Goldberg, A. L. (2001) J Biol Chem 276, 24261-24267

13. Pfluger, K., and Muller, V. (2004) Journal of bioenergetics and biomembranes 36, 17-24

14. Scire, A., Marabotti, A., Aurilia, V., Staiano, M., Ringhieri, P., Iozzino, L., Crescenzo, R., Tanfani, F., and D'Auria, S. (2008) Proteins 73, 839-850

15. Zaccai, G. (2000) Science 288, 1604-1607

16. Zaccai G. (2013). Current Physical Chemistry, Special Issue on Quantum Nanobiology and Biophysical Chemistry, K J Jalkanen, Ed 3(1): 9-16.

17. Tehei, M., Franzetti, B., Madern, D., Ginzburg, M., Ginzburg, B. Z., Giudici-Orticoni, M. T., Bruschi, M., and Zaccai, G. (2004) EMBO Rep 5, 66-70

18. Tehei, M., Madern, D., Franzetti, B., and Zaccai, G. (2005) J Biol Chem 280, 40974-40979

19. Colletier, J. P., Aleksandrov, A., Coquelle, N., Mraihi, S., Mendoza-Barbera, E., Field, M., and Madern, D. (2012) Mol Biol Evol

20. Macario, A. J., Lange, M., Ahring, B. K., and De Macario, E. C. (1999) Microbiol Mol Biol Rev 63, 923-967, table of contents.

21. Mota, M. J., Lopes, R. P., Delgadillo, I., and Saraiva, J. A. (2013) Biotechnol Adv

22. Oger, P. M., and Cario, A. (2013) Biophys Chem

23. Coker, J. A., DasSarma, P., Kumar, J., Muller, J. A., and DasSarma, S. (2007) Saline systems 3,6

24. Franzetti, B., Schoehn, G., Ebel, C., Gagnon, J., Ruigrok, R. W., and Zaccai, G. (2001) J Biol Chem 276, 29906-29914.

25. Takai, K., and Nakamura, K. Curr Opin Microbiol 14, 282-291

26. Zierenberg, R. A., Adams, M. W., and Arp, A. J. (2000) Proc Natl Acad Sci U S A 97 , 12961-12962

27. Jackson, R. B., Carpenter, S. R., Dahm, C. N., McKnight, D. M., Naiman, R. J., Postel, S. L., and Running, S. W. (2001) Ecol Appl 11, 1027-1045

28. McCready, S., Muller, J. A., Boubriak, I., Berquist, B. R., Ng, W. L., and DasSarma, S. (2005) Saline systems 1,3 
29. Baliga, N. S., Bjork, S. J., Bonneau, R., Pan, M., Iloanusi, C., Kottemann, M. C., Hood, L., and DiRuggiero, J. (2004) Genome Res 14, 1025-1035

30. Whitehead, K., Kish, A., Pan, M., Kaur, A., Reiss, D. J., King, N., Hohmann, L., DiRuggiero, J., and Baliga, N. S. (2006) Molecular systems biology 2, 47

31. Fendrihan, S., Berces, A., Lammer, H., Musso, M., Ronto, G., Polacsek, T. K., Holzinger, A., Kolb, C., and Stan-Lotter, H. (2009) Astrobiology 9, 104-112

32. Kottemann, M., Kish, A., Iloanusi, C., Bjork, S., and Diruggiero, J. (2005) Extremophiles 9, 219-227

33. Moissl-Eichinger, C. The ISME journal 5, 209-219

34. Moissl, C., Bruckner, J. C., and Venkateswaran, K. (2008) The ISME journal 2, 115-119

35. McGenity, T. J., Gemmell, R. T., Grant, W. D., and Stan-Lotter, H. (2000) Environ Microbiol 2, 243-250

36. Gramain, A., Diaz, G. C., Demergasso, C., Lowenstein, T. K., and McGenity, T. J. Environ Microbiol

37. Grant, W. D., Gemmell, R. T., and McGenity, T. J. (1998) Extremophiles 2, 279-287

38. Schubert, B. A., Lowenstein, T. K., Timofeeff, M. N., and Parker, M. A. Environ Microbiol $12,440-454$

39. Vreeland, R. H., Rosenzweig, W. D., and Powers, D. W. (2000) Nature 407, 897-900

40. Tehei, M., Franzetti, B., Maurel, M. C., Vergne, J., Hountondji, C., and Zaccai, G. (2002) Extremophiles 6, 427-430

41. Hegde, S., and Kaltenegger, L. (2013) Astrobiology 13, 47-56

42. Kounaves, S. (2007) Nature 449, 281

43. Andrews-Hanna, J. C., Phillips, R. J., and Zuber, M. T. (2007) Nature 446, 163-166

44. Landis, G. A. (2001) Astrobiology 1, 161-164

45. Marion, G. M., Fritsen, C. H., Eicken, H., and Payne, M. C. (2003) Astrobiology 3, 785-811

46. Daffonchio, D., Borin, S., Brusa, T., Brusetti, L., van der Wielen, P. W., Bolhuis, H., Yakimov, M. M., D'Auria, G., Giuliano, L., Marty, D., Tamburini, C., McGenity, T. J., Hallsworth, J. E., Sass, A. M., Timmis, K. N., Tselepides, A., de Lange, G. J., Hubner, A., Thomson, J., Varnavas, S. P., Gasparoni, F., Gerber, H. W., Malinverno, E., Corselli, C., Garcin, J., McKew, B., Golyshin, P. N., Lampadariou, N., Polymenakou, P., Calore, D., Cenedese, S., Zanon, F., and Hoog, S. (2006) Nature 440, 203-207

47. Borin, S., Brusetti, L., Mapelli, F., D'Auria, G., Brusa, T., Marzorati, M., Rizzi, A., Yakimov, M., Marty, D., De Lange, G. J., Van der Wielen, P., Bolhuis, H., McGenity, T. J., Polymenakou, P. N., Malinverno, E., Giuliano, L., Corselli, C., and Daffonchio, D. (2009) Proc Natl Acad Sci U S A 106, 9151-9156

48. $\quad$ Knauth, L. P. (1998) Nature 395, 554-555

49. Ginzburg, M., Sachs, L., and Ginzburg, B. Z. (1970) J Gen Physiol 55, 187-207.

50. Oren, A., Heldal, M., Norland, S., and Galinski, E. A. (2002) Extremophiles 6, 491-498

51. Lanyi, J. K., and Silverman, M. P. (1972) Canadian journal of microbiology 18, 993-995

52. Grant, W. D. (2004) Philosophical transactions of the Royal Society of London 359, 12491266; discussion 1266-1247

53. Lanyi, J. K. (1974) Bacteriological reviews 38, 272-290

54. Madern, D., Ebel, C., and Zaccai, G. (2000) Extremophiles 4, 91-98

55. Jasnin, M., Moulin, M., Haertlein, M., Zaccai, G., and Tehei, M. (2008) EMBO Rep 9, 543547

56. Stadler, A. M., Embs, J. P., Digel, I., Artmann, G. M., Unruh, T., Buldt, G., and Zaccai, G. (2008) Journal of the American Chemical Society 130, 16852-16853

57. Tehei, M., Franzetti, B., Wood, K., Gabel, F., Fabiani, E., Jasnin, M., Zamponi, M., Oesterhelt, D., Zaccai, G., Ginzburg, M., and Ginzburg, B. Z. (2007) Proc Natl Acad Sci U $S$ A 104, 766-771

58. Persson, E., and Halle, B. (2008) Proc Natl Acad Sci U S A 105, 6266-6271

59. Capes, M. D., DasSarma, P., and DasSarma, S. (2012) BMC genomics 13, 39 
60. Costenaro, L., Zaccai, G., and Ebel, C. (2002) Biochemistry 41, 13245-13252

61. Irimia, A., Ebel, C., Madern, D., Richard, S. B., Cosenza, L. W., Zaccai, G., and Vellieux, F. M. (2003) J Mol Biol 326, 859-873

62. Deole, R., Challacombe, J., Raiford, D. W., and Hoff, W. D. (2013) J Biol Chem 288, 581588

63. Oren, A. (2013) FEMS Microbiol Lett 342, 1-9

64. Coquelle, N., Talon, R., Juers, D. H., Girard, E., Kahn, R., and Madern, D. J Mol Biol 404, 493-505

65. Ebel, C., Faou, P., Kernel, B., and Zaccai, G. (1999) Biochemistry 38, 9039-9047.

66. Zaccai, G. (2011) J. Non-Cryst. Solids 357, 615-621

67. Marty, V., Jasnin, M., Fabiani, E., Vauclare, P., Gabel, F., Trapp, M., Peters, J., Zaccai, G., and Franzetti, B. (2013) Journal of the Royal Society, Interface / the Royal Society 10, 20130003

68. Kuo, Y. P., Thompson, D. K., St. Jean, A., Charlebois, R. L., and Daniels, C. J. (1997) J Bacteriol 179, 6318-6324

69. Chamieh, H., Marty, V., Guetta, D., Perollier, A., and Franzetti, B. (2012) Extremophiles $16,215-225$

70. Chamieh, H., Guetta, D., and Franzetti, B. (2008) Biochem J 411, 387-397

71. Morimoto, R. I., and Cuervo, A. M. (2009) The journals of gerontology 64, 167-170

72. Maurizi, M. R., and Xia, D. (2004) Structure (Camb) 12, 175-183

73. Smith, D. M., Benaroudj, N., and Goldberg, A. (2006) J Struct Biol 156, 72-83 